Repository of the Max Delbrück Center for Molecular Medicine (MDC) Berlin (Germany)

http://edoc.molc-berlin.de/13807/

\title{
(Pro)renin receptor and V-ATPase: from Drosophila to humans
}

Rousselle, A., Sihn, G., Rotteveel, M., Bader, M. 


\section{(Pro)renin receptor and V-ATPase: From Drosophila to Man}

Anthony Rousselle ${ }^{1}$, Gabin Sihn ${ }^{1}$, Martijn Rotteveel ${ }^{1}$, and Michael Bader ${ }^{1,2,3}$

${ }^{1}$ Max-Delbrück-Center for Molecular Medicine (MDC), Berlin-Buch, Germany

${ }^{2}$ Charité-University Medicine, Berlin, Germany

${ }^{3}$ University of Lübeck, Germany

Corresponding author

Prof. Dr. Michael Bader

Max-Delbrück-Center for Molecular Medicine

Robert-Rössle-Str. 10,

D-13125 Berlin-Buch, Germany

Email: mbader@mdc-berlin.de

Tel: +493094062193

Fax: +49 3094062110 


\begin{abstract}
A decade ago, the (pro)renin receptor ((P)RR) was discovered and depicted as a potential activator of the tissue renin-angiotensin system. For this reason, the role of the (P)RR in cardiovascular diseases and diabetes has been particularly studied. However, the discovery of embryonic lethality after (P)RR gene deletion in mouse and zebrafish paved the way for additional roles of (P)RR in cell homeostasis. Indeed, the (P)RR has been shown to associate with the vacuolar $\mathrm{H}^{+}$-ATPase, hence its other name ATP6ap2. Developmental studies in Xenopus and Drosophila have revealed an essential role of this association to promote the canonical and non-canonical Wnt signaling pathways, while studies with tissue specific gene deletion have pointed out a role in autophagy. This review aims to summarize recent findings on the cellular functions of (P)RR emerging from various mutated and transgenic animal models.
\end{abstract}

Key words: V-ATPase, Wnt signaling, autophagy, endocytosis, acidification.

Abbreviations: Ang I, angiotensin I; Ang II, angiotensin II; ER, endoplasmic reticulum; Fmi, Flamingo; Fz, Frizzled; HRP, handle region peptide; KO, knockout; LRP6, low-density lipoprotein receptor-related protein 6; MAPK, mitogen-activated protein kinase; MVBs, multivesicular bodies; PCP, planar cell polarity; pn, pekin; (pro)renin, prorenin and renin; (P)RR, (pro)renin receptor; RAS, renin-angiotensin system; RNAi, RNA interference; siRNA, small interfering RNA; s(P)RR, soluble (P)RR; V-ATPase, vacuolar $\mathrm{H}^{+}$-ATPase; XLMR, X-linked mental retardation; XPDS, X-linked Parkinsonism with spasticity. 


\section{INTRODUCTION}

For many years, the renin-angiotensin system (RAS) has been described as a circulating system essential for the regulation of blood pressure and salt homeostasis. Within the enzymatic cascade leading to angiotensin II (AngII) generation, renin controls the ratelimiting conversion of circulating angiotensinogen into angiotensin I (AngI). The common view was that renin was biologically active only after its proteolytic activation in juxtaglomerular cells of the kidney and its secretion into the blood circulation. Its precursor prorenin was believed to have no functional relevance. The emergence of a tissue RAS with the local production of AngII and the discovery of several new components have challenged these dogma [1]. In 2002, Nguyen et al. provided confirmatory evidence for concept with the discovery of a membrane-bound protein that could bind both prorenin and renin ((pro)renin) [2] (Figure 1). The protein was called (pro)renin receptor ((P)RR) and differed from other described (pro)renin binding sites by its ability, upon (pro)renin binding, to trigger cellular responses.

Upon binding to $(\mathrm{P}) \mathrm{RR}$, first, the enzymatic activity of prorenin and renin was enhanced, resulting in increased AngI production and the subsequent generation of AngII at the tissue level. In addition, activation of (P)RR triggered intracellular activation of several Mitogenactivated protein kinases (MAPK) and led to the secretion of inflammatory and pro-fibrotic molecules in various cell types ([2]. As these latter signaling events occurred independently of the generation of AngII, it was suggested that (pro)renin-bound (P)RR might promote direct tissue damage and contribute to the pathogenesis of cardiovascular diseases by assisting in the activation of the local RAS. Thus, (P)RR directly became an interesting target to decrease end-organ damage associated with hypertension and diabetes and to complement the arsenal of anti-hypertensive drugs, which do not fully prevent these tissue damages.

In 2004, a (P)RR blocker susceptible to interfere with prorenin/(P)RR interaction, was shown to decrease renal AngII levels and to prevent the development of nephropathy in diabetic rats [3]. Despite these encouraging results, conflicting data were obtained in various animal models and raised a controversy about the binding ability, specificity and properties of this blocker. These aspects have been extensively reviewed and will not be further developed here [4-6].

The gene coding for the $(\mathrm{P}) \mathrm{RR}$ is located on the $\mathrm{X}$ chromosome and codes for a protein with a length of 350 amino acids and an approximate mass of $37 \mathrm{kDa}$. Structural analysis of (P)RR has shown that it contains two hydrophobic domains predicted to be a signal peptide, and a transmembrane domain suggesting a type I transmembrane protein [7]. Thus, (P)RR can be divided into four domains: an N-terminal signal peptide, an extracellular domain binding (pro)renin, a single transmembrane domain and a short cytoplasmic domain (Figure 2) [8]. In addition, shedding by proteases such as furin or ADAM19 allows the generation of a long soluble fragment of 28-29 kDa composed of the (pro)renin binding domain (s(P)RR) and a short fragment composed of the transmembrane and intracellular domain $([9,10]$. The fulllength $(\mathrm{P}) \mathrm{RR}$ seems to be ubiquitously expressed but in vivo data about the genesis and the regulation of soluble $(\mathrm{P}) \mathrm{RR}$ are missing.

Few years after (P)RR was discovered, and in parallel to its characterization as a receptor playing a role within the RAS, new evidence emerged, which would drastically change our vision on this protein and its function. In the paper by Nguyen et al. [2], (P)RR had been described to be partially identical to a protein identified as early as 1998 (Figure 1). In chromaffin cells of the adrenal medulla, Ludwig et al. [11] had described a truncated form (called M8-9) of the (P)RR roughly corresponding to the short fragment generated after shedding $[12,13]$. This M8-9 portion was found to co-immunoprecipitate with the V-ATPase which would later urge the scientific community to rename (P)RR as V-ATPase-associated 
protein 2 (ATP6ap2). The V-ATPase is a multi-subunit complex composed of a peripheral V1 domain (8 subunits) responsible of ATP hydrolysis and a V0 domain (6 subunits) responsible for proton translocation [14]. In addition, accessory subunits have been described, Ac45 (ATP6ap1) and -hence- ATP6ap2, although this denomination has yet to be proven at the functional level. In most cells, V-ATPases are mainly located in intracellular compartments such as endosomes and lysosomes, among others. These proton pumps provide an acidic environment in the lumen of these vesicles essential for protein trafficking (endocytosis and exocytosis), maturation, recycling and degradation through $\mathrm{pH}$-dependent enzymes $[14,15]$. Also, in some specific cells, V-ATPases are targeted to the plasma membrane like in osteoclasts (to resorb bone) and in intercalated cells of the renal collecting duct (to acidify urine). Deletion or mutation of V-ATPase subunits in animal models and humans are associated to severe phenotypes including early death [16, 17].

In light of the fact that (P)RR could be part of the V-ATPase complex, it has not been surprising that $(\mathrm{P}) \mathrm{RR}$ was mainly found to be localized intracellularly, in contrast to its original description at the cell surface. Such intracellular localization has been since then substantiated by immunofluorescence and biochemical studies [7, 9, 10, 18-20]. In addition, sequence analysis has revealed two putative motifs in the cytoplasmic domain of (P)RR which further predict its targeting to distinct intracellular compartments [7, 12].

Thus, a few years after its discovery, as receptor for (pro)renin, it was suggested that (P)RR might play an additional role in association with the V-ATPase. But at that time, direct evidence was lacking and the research field was focused on the ability of (P)RR to stimulate the tissue RAS. Study with mutant zebrafish and the impossibility to generate (P)RR knockout (KO) mice suggested a more essential role of this protein than the RAS (Figure 1), thus engendering a progressive, yet irresistible, shift of the (P)RR research towards critical intracellular functions. However, additional years were necessary to conduct developmental studies and generate mice with tissue-specific deletion of (P)RR in order to unravel such functions. This review will summarize advances provided from various animal models and what is known in humans. 


\section{ZEBRAFISH}

Studies with zebrafish provided early hints for a potential role of (P)RR as a V-ATPase accessory subunit. Indeed, zebrafish with insertional mutagenesis of (P)RR shared a common embryonic phenotype with mutants for several V-ATPase subunits (Figure 1). All mutants displayed abnormal pigmentation, multiple organ defects and necrosis in the central nervous system, finally resulting in lethality after 5 days of development [21, 22] (http://zfin.org). We obtained the same results using morpholino antisense oligonucleotide injections against (P)RR in zebrafish (Sihn et al., manuscript in preparation). These results suggested that (P)RR is important for embryonic development probably linked to V-ATPase activity but no direct evidence was provided by this animal model for years. Recently, Eauclaire et al. performed a chemical mutagenesis screen to identify proteins important for biliary development [23]. Among all mutants with abnormalities in hepatic development, one mutant called pekin ( $p n$ ) was particularly studied. It turned out that the causative mutation in $p n$ was located at the end of exon 8 of the atp6ap2 gene and led to a read-through and premature stop codon. Ultrastructural examination of bile duct cells revealed accumulation of electron dense bodies at the periphery of the cells. In addition, typical features of endoplasmic reticulum (ER) stress were also present. Thus, it was suggested that decreased ATP6ap2 levels altered protein trafficking and degradation probably by affecting V-ATPase function and altering vesicular $\mathrm{pH}$. The presence of skin and eye hypopigmentation in $p n$ provided further evidence as this phenotype was previously associated with inhibition of the V-ATPase [21, 22, 24, 25]. In the skin, melanoblast markers decreased with time which suggests that melanocytes developed normally but underwent degeneration [23]. In the eye, striking degradation of various cell layers was also observed. The fact that mutants for ATP6V0D1 and ATP6ap1 displayed defects in intrahepatic ducts similar to pn, and that renin morphant displayed minor defects suggested that ATP6ap2 is important for V-ATPase activity independently of its (pro)renin receptor ability. Further studies are necessary to reveal the exact mechanism for which (P)RR is essential in biliary development. 


\section{XENOPUS}

In 2010, the group of C. Niehrs carried out a genome-wide siRNA screen in order to identify new members of the $\mathrm{Wnt} / \beta$-catenin signaling pathway [26]. Wnts are a superfamily of secreted glycoproteins which signal through various receptors and co-receptors. The so-called canonical Wnt signaling involves $\beta$-catenin as a major downstream effector and is essential for embryonic development and cellular growth. Impaired Wnt signaling is associated with various human diseases including carcinogenesis [27]. The initiation of the Wnt/ $\beta$-catenin signaling requires the formation of a tertiary complex composed of a soluble Wnt ligand, the receptor Frizzled (Fz) and low-density lipoprotein receptor-related protein 6 (LRP6) as a coreceptor. This leads to the stabilization of $\beta$-catenin, which translocates into the nucleus and induces transcription of downstream genes [28]. An important step in this cascade is the aggregation of LRP6 and the formation of a signalosome at the plasma membrane [29]. Following LRP6 phosphorylation, the complex is internalized in vesicle and subsequent endocytosis is essential to transduce the signal [28, 30, 31].

From their screen, Cruciat et al. revealed that (P)RR is important to initiate the canonical Wnt signaling pathway [26] (Figure 1). Absence of (P)RR resulted in abnormal frog embryos with small heads, shortened tails and hypopigmentation as described in (P)RR mutant zebrafish embryos [21, 22] (http://zfin.org). In addition, a loss of anterior-posterior neural patterning was also observed. They showed that (P)RR was required for V-ATPase-mediated signalosome acidification and subsequent LRP6 phosphorylation [26] (Figure 3). Indeed, decreased acidification following inhibition of V-ATPase by RNAi and pharmacological treatment, impaired LRP6 phosphorylation and Wnt signaling in two different cell lines, and mimicked results obtained after deletion of (P)RR. At the molecular level, (P)RR was described to provide a bridge between the Wnt receptor complex and the V-ATPase. Interestingly, the extracellular domain of the (P)RR was sufficient to bind Fz8 and LRP6 (Figure 2) but the transmembrane domain was necessary to transduce the signal. On the contrary, both domains were required to bind (directly or indirectly) ATP6V0C and ATP6V0D1 (Figure 2). Thus, the cytoplasmic part of the (P)RR which is important for signal transduction after (pro)renin binding does not seem to play a role here. Also, it is important to stress that prorenin is absent at early stages of development and renin addition did not influence the Wnt reporter assay. Altogether, this study was the first to provide an explanation for the essential role of (P)RR for normal embryonic development, independently of its ability to bind (pro)renin. 


\section{DROSOPHILA}

In addition to the canonical Wnt signaling described above, two additional noncanonical pathways are also existing and known as $\mathrm{Wnt} / \mathrm{Ca}^{2+}$ and the planar cell polarity (PCP) pathway. The latter involves Fz receptors and the downstream activation of small GTPases inducing expression of their target genes and changes in cytoskeleton dynamics [28]. The PCP is a highly conserved pathway essential to orient and polarize cells in the plane of a tissue. In Drosophila, PCP is important for the orientation of hairs and bristles of the wing among other structures. Two independent genome-wide RNA interference (RNAi) screens in Drosophila revealed an important role of the gene CG8444 in the canonical Wnt signaling [32] and the PCP pathway [33]. This gene codes for a transmembrane protein which was originally named VhaM8-9 due to its sequence homology to ATP6ap2 but whose function was not fully characterized [34]. In the two novel studies, to refer to its role as a receptor of (pro)renin, it was renamed to dPRR (Drosophila homolog of prorenin receptor) by Buechling et al. and to VhaPRR (vacuolar-type $\mathrm{H}^{+}$-ATPase prorenin receptor) by Hermle et al. [35, 36]. These studies in Drosophila concordantly described a role of (P)RR in the PCP pathway [35, 36] (Figures 1 and 3). siRNA against (P)RR resulted in severe PCP defects, characterized by abnormal anterior-posterior orientation and hair mispolarization. Interestingly, the phenotype could be rescued by injection of the full-length human (P)RR mRNA but not with a Nterminally truncated form [35, 36]. This suggested an essential role of the extracellular domain to transduce the noncanonical Wnt signaling, in a fashion similar to what has been described for the canonical signaling [26]. In addition, (P)RR was shown to interact with Fz (Figure 2), and the lack of (P)RR impaired targeting of $\mathrm{Fz}$ to the plasma membrane and thus disrupted its asymmetric localization necessary for normal pupal wings [35, 36]. In contrast, components of the canonical signaling (Fz2 receptor and coreceptor arrow) displayed a normal localization [36]. Thus, it was suggested that (P)RR, through potential regulation of V-ATPase, was important for PCP initiation by trafficking specifically Fz to the plasma membrane. To study this functional association, RNAi against $12 \mathrm{~V}$-ATPase subunits were tested. Surprisingly, only the deletion of the subunit B mimicked the severe defects observed after (P)RR ablation [36]. Thus, the exact mechanism remained obscure and requires further analysis. The absence of a renin/prorenin system in Drosophila suggests that this mechanism does not involve the ability of (P)RR to bind (pro)renin.

In an effort to better understand the role of (P)RR within the PCP pathway, the group of $\mathrm{M}$. Simons generated mutant flies with clonal deletion of (P)RR in the pupal wing [37]. In epithelial cells, an important feature of the PCP signaling is the formation of asymmetric PCP domains at apical junctions. To date, six proteins including $\mathrm{Fz}$ and Flamingo (Fmi) were described to form these structures and are termed PCP core proteins. This study showed that clonal elimination of (P)RR led to strong PCP defects consistent with the previous reports $[35,36]$ but it also provided new molecular mechanisms for (P)RR (Figure 1). Indeed, (P)RR was shown to co-localize with PCP core proteins during all stages of pupal wing development [37]. Absence of (P)RR reduced the presence of PCP proteins such as Fz and Fmi at apical junctions of cells (Figure 3). Instead, these proteins were seen to localize in vesicular compartments. Previously described to bind Fz, the extracellular domain of (P)RR interacted also with Fmi in this model and thus, was important for normal targeting of both proteins (Figures 2 and 3). Interestingly, the cleavage site and hence the soluble (P)RR were not required for normal PCP signaling as rescue of mutant flies with a construct expressing a noncleavable (P)RR restored all aspects of the phenotype. A reciprocal association was also revealed with an essential role of Fmi to recruit (P)RR to the PCP domain and for subsequent apical trafficking. Altogether, these results suggested that (P)RR possessed all the characteristics of a PCP core protein and may be considered as such. It is important to note that genetic manipulation of several V-ATPase subunits did not impair PCP defects nor 
asymmetric localization of PCP core proteins. However, one substantial difference with mutants for PCP core proteins was that lack of (P)RR significantly affected cell viability. It suggested that $(\mathrm{P}) \mathrm{RR}$ might play an additional role due to its association with the V-ATPase [37]. Using elegant experiments based on endocytosis quantification and $\mathrm{pH}$ monitoring, they showed that $(\mathrm{P}) \mathrm{RR}$ regulated acidification of specific apical vesicles but did not interfere with other vesicle populations. Defective apical vesicles led to a mistrafficking of the transmembrane protein E-cadherin which could not transit through the endolysosomal pathway and undergo lysosomal degradation. Instead, after normal endocytosis, E-Cadherin was recycling back to the apical membrane. Thus it appeared that (P)RR had a specific role for recycling apical vesicles in epithelial cells (Figures 1 and 3). A very important discovery was also that V-ATPase-mediated acidification of certain compartments did not require (P)RR. In contrast, V-ATPase was required for acidification of all vesicles as mutant flies for Vha6-2 (ATP6V1A) showed impaired endocytosis and subsequent acidification at the apical and basal areas.

Altogether, this study unrevealed that (P)RR and V-ATPase shared overlapping role in the endolysosomal pathway but exhibit distinct functions in the PCP pathway. 


\section{MOUSE}

Our early attempts to generate a (P)RR deficient mouse were unsuccessful as no chimeras were obtained after implantation of blastocysts containing (P)RR deficient embryonic stem cells into foster mothers [17]. This suggested that the complete deletion of (P)RR was lethal (Figure 1). Although embryonic lethality could not be ascertained, this was reminiscent of the early death observed in mutant zebrafish. Nevertheless, such early lethality in the mouse would rule out a function of the (P)RR solely as a component of the RAS. Indeed, mice deficient for various RAS components (renin, angiotensinogen...) develop normally [38]. Thus, it was suggested that this essential role of (P)RR should involve an additional function independently of the RAS.

To circumvent this early death, conditional deletions of (P)RR in mice using the Cre/Lox system were developed (Figure 1). The first tissue-specific deletion of (P)RR in mouse confirmed the potential influence of (P)RR on V-ATPase function in particular in the process of autophagy (Figure 3). Autophagy is a general term for an essential cellular process allowing the cell to degrade organelles and cytosolic proteins. Autophagy plays important physiological roles for adaptation to starvation, development, elimination of pathogens and cell death. This non-specific process is initiated with the engulfment of a portion of cytoplasm within a specific organelle called autophagosome [39, 40]. These engulfed components are degraded when this structure further fuses with lysosomes containing $\mathrm{pH}$-dependent hydrolases. The V-ATPase plays a fundamental role within this process by generating the acidic $\mathrm{pH}$ necessary for hydrolase activity.

Mice with a specific deletion of (P)RR in cardiomyocytes developed abnormal heart parameters resulting in heart failure and died within 3 weeks after birth [41]. Histologic observations revealed vast fibrotic areas and degenerating cardiomyocytes exhibiting perinuclear accumulation of autophagic vacuoles. Similar observations were obtained for mice with a conditional deletion of (P)RR in podocytes which developed nephrotic syndrome and fulminant kidney failure leading to death within 2-4 weeks after birth [42, 43]. Podocyte death was also associated with an accumulation of undigested proteins in autophagic vacuoles and enhanced ER stress. In addition, these cells exhibited features of a more global dysfunction with altered cytoskeleton, loss of differentiation markers and evidence of necrosis. These results were mimicked in vitro in embryonic fibroblasts [41] and podocytes $[42,43]$ after treatment with bafilomycin. In addition, (P)RR ablation in these cells in culture induced a downregulation of several subunits of the V0 sector of the V-ATPase and impaired acidification [41-43]. Such impairment in autophagy is reminiscent of the phenotype observed in yeast lacking a single V0 subunit or proteins involved in the V-ATPase assembly [44, 45]. Altogether, these studies in the mouse have suggested that the lack of (P)RR may impact on V-ATPase assembly, stability or expression. However, the exact mechanism is still unknown and further investigations are necessary. 


\section{HUMAN}

Genome-wide association studies with Japanese cohorts have revealed various polymorphisms in the $(P) R R / A T P 6 a p 2$ gene associated with increased cardiovascular risks in humans [6]. Firstly, the 5+169C $>$ T polymorphism was significantly associated to blood pressure in Japanese men [46] and more recently also in Caucasian men [47]. Secondly, the $+1513 \mathrm{~A}>\mathrm{G}$ polymorphism was significantly associated with the risk of lacunar infarction and left ventricular hypertrophy in Japanese women [48]. Finally, two additional polymorphisms were significantly associated with hypertension in additional populations with vascular diseases [49]. However, none of these reports provided evidence of an impact of these polymorphisms on (P)RR/ATP6ap2 expression or function.

In fact, the most interesting clinical aspect has been provided by Ramser et al. in 2005. This study identified a unique exonic splice enhancer mutation (c.321C $>$ T) resulting in the deletion of the exon $4(\Delta \mathrm{e} 4$ isoform) and in a reduction of $50 \%$ in the amount of the functional protein [50]. This mutation does not lead to cardiovascular abnormalities but results in X-linked mental retardation (XLMR) and epilepsy (Figure 1). Interestingly, the authors still linked this phenotype to the RAS: indeed, experiments conducted on the lymphocytes of one patient bearing the mutation, showed that the $\Delta \mathrm{e} 4$ isoform was unable to activate ERK $1 / 2$ in the presence of renin. This result suggested that the function as a receptor for renin might be involved in neuron physiology [50].

A recent study found the same mutation in a patient with another X-linked mental disease [51]. In addition, the authors described a new mutation c.345C $>\mathrm{T}$ associated with $\mathrm{X}$-linked Parkinsonism with spasticity (XPDS). This mutation also resulted in exon 4 skipping and overexpression of the minor splice $\Delta \mathrm{e} 4$ isoform. In HEK293 cells transfected with siRNA, (P)RR ablation impaired autophagy and lysosomal clearance as observed in XPDS brain sections. Of note, the most drastic effects were found in the striatum, a region involved in Parkinson's disease, and associated with an accumulation of Tau protein [52]. Interestingly, transfection of the $\Delta \mathrm{e} 4$ isoform into (P)RR-depleted HEK293 cells did not significantly influence the canonical Wnt signaling pathway as assessed by luciferase reporter assay [51].

This study confirmed that (P)RR mutations are associated with various mental diseases but the described mechanism differed from the initial study [50]. Even though (pro)renin binding activity of (P)RR was not excluded, the defective autophagy strongly argued for a role of (P)RR as regulator of V-ATPase activity. This conclusion was in line with the work of Contrepas et al. which showed that the truncated (P)RR/ATP6ap2 acted as a dominant negative mutant in transfected neuronal cells by affecting the trafficking of the endogenous receptor [53]. Because only one copy of (P)RR/ATP6ap2 is present per synaptic vesicle [54], it was suggested that intact (P)RR may be important for vesicle trafficking and/or recycling and more generally for neurotransmission. In light of the recent findings, we can assume that this impaired neurotransmission might be a consequence of abnormal cell homeostasis due to impaired autophagy and vesicular acidification. 


\section{CONCLUSIONS AND PERSPECTIVES}

From its initial description as a receptor for (pro)renin in 2002, the understanding of (P)RR has markedly evolved. It got named ATP6ap2 and an essential role as a modulator of VATPase activity was suggested (Figure 1). Accordingly, several animal models have confirmed that (P)RR is important for canonical Wnt and PCP signaling, autophagy and vesicular trafficking, all processes in which the V-ATPase is also involved (Figure 2). However, the exact mechanism how (P)RR regulates the V-ATPase is still obscure.

Multivesicular bodies (MVBs) also known as multivesicular endosomes are structures sequestering early and late endosomes before lysosomal degradation [14, 55]. MVBs have been described to be essential for the transduction of canonical Wnt signaling [56] but also to generate exosomes containing Wnt proteins [57]. Several studies reported that V-ATPase regulates the function of MVBs [57-59]. Thus, it would be interesting to investigate whether (P)RR is important for the generation and maturation of MVBs.

Another important aspect is that (P)RR and V-ATPase are not always working in concert to regulate trafficking and acidification in epithelial cells [37]. Thus, it is essential to decipher in which mechanisms (P)RR is important for V-ATPase activity. Also, the role of (P)RR described in polarized cells has to be confirm in other cell types. In addition, growing evidence suggest that the V0 domain of the V-ATPase orchestrates membrane fusion independently of its proton pump activity during synaptic exocytosis, osteoclastogenesis and phagocytosis [60-62]. A recent study in yeast has suggested that a specific conformation of the V0 subunits is required for proper vacuole fusion [63]. Because membrane fusion is important for autophagolysosome formation and vesicle endo/exocytosis, it is tempting to speculate that (P)RR might be important not only for acidification, but also for membrane fusion. This potential dual function of $(\mathrm{P}) \mathrm{RR}$ has yet to be proven and certainly requires additional studies.

The exact role and regulation of the $s(P) R R$ is also still unclear. It was described that $s(P) R R$ can be secreted by cells into the blood stream [9] and this aspect initiated the development of enzyme-linked immunosorbent assays to detect and quantify s(P)RR in various biological fluids [4, 64]. From developmental studies, it was shown that s(P)RR can bind to Fz and LRP6 in in vitro assay but could not rescue Wnt signaling after (P)RR depletion [26]. Also, $\mathrm{s}(\mathrm{P}) \mathrm{RR}$ was described to be endocytosed together with Fmi in epithelial cells but without any relevance for PCP signaling or cell viability [37]. Accordingly, the meaning of such interactions in vivo is totally unknown.

Finally, as a new member of the local RAS, targeting the extracellular part to inhibit the (pro)renin/(P)RR binding was very tempting until few years ago. However, the lack of in vivo evidence that this interaction triggers signaling pathways independently of the generation of Ang II, the ongoing controversy on the efficiency of the putative (P)RR blocker (HRP) and last but not least, the essential role of (P)RR in cell physiology, will probably put a brake on this idea. If not, one should carefully evaluate potential blockers to exclude any adverse effects due to impairment of V-ATPase function. For instance, a recent study showed that in vitro, HRP could act as a partial agonist and stimulate V-ATPase activity in a (P)RRdependent manner [65]. Further knowledge about the exact contribution of the extracellular part to the V-ATPase activity will probably precede any drug design and blocking strategy. 
After 10 years of intensive research, the role of $(\mathrm{P}) \mathrm{RR}$ as a regulator of V-ATPase function is now well described but there are still a lot of questions about its role in cellular and physiological functions.

\section{ACKNOWLEDGEMENTS}

The work of the authors on (P)RR was funded by the Deutsche Forschungsgemeinschaft (BA1374/20-1). M.R. was supported by fellowships of the Dutch Ministry of Education, Culture and Science and from the Erasmus program of the European Commission. 


\section{REFERENCES}

1. Bader, M. (2010). Tissue renin-angiotensin-aldosterone systems: Targets for pharmacological therapy. Annu Rev Pharmacol Toxicol 50, 439-465.

2. Nguyen, G., Delarue, F., Burckle, C., Bouzhir, L., Giller, T., and Sraer, J.D. (2002). Pivotal role of the renin/prorenin receptor in angiotensin II production and cellular responses to renin. J Clin Invest 109, 1417-1427.

3. Ichihara, A., Hayashi, M., Kaneshiro, Y., Suzuki, F., Nakagawa, T., Tada, Y., Koura, Y., Nishiyama, A., Okada, H., Uddin, M.N., et al. (2004). Inhibition of diabetic nephropathy by a decoy peptide corresponding to the "handle" region for nonproteolytic activation of prorenin. J Clin Invest 114, 1128-1135.

4. Ichihara, A., Sakoda, M., Kurauchi-Mito, A., Narita, T., Kinouchi, K., Murohashi-Bokuda, K., and Itoh, H. (2010). Possible roles of human (pro)renin receptor suggested by recent clinical and experimental findings. Hypertens Res 33, 177-180.

5. Nguyen, G. (2011). Renin and prorenin receptor in hypertension: what's new? Current hypertension reports $13,79-85$.

6. Krop, M., Lu, X., Danser, A.H., and Meima, M.E. (2013). The (pro)renin receptor. A decade of research: what have we learned? Pflugers Arch 465, 87-97.

7. Sihn, G., Burckle, C., Rousselle, A., Reimer, T., and Bader, M. (2013). (Pro)renin receptor: subcellular localizations and functions. Frontiers in bioscience (Elite edition) 5, 500-508.

8. Nguyen, G., and Contrepas, A. (2008). Physiology and pharmacology of the (pro)renin receptor. Current opinion in pharmacology 8, 127-132.

9. Cousin, C., Bracquart, D., Contrepas, A., Corvol, P., Muller, L., and Nguyen, G. (2009). Soluble form of the (pro)renin receptor generated by intracellular cleavage by furin is secreted in plasma. Hypertension 53, 1077-1082.

10. Yoshikawa, A., Aizaki, Y., Kusano, K., Kishi, F., Susumu, T., lida, S., Ishiura, S., Nishimura, S., Shichiri, M., and Senbonmatsu, T. (2011). The (pro)renin receptor is cleaved by ADAM19 in the Golgi leading to its secretion into extracellular space. Hypertens Res 34, 599-605.

11. Ludwig, J., Kerscher, S., Brandt, U., Pfeiffer, K., Getlawi, F., Apps, D.K., and Schagger, H. (1998). Identification and characterization of a novel 9.2-kDa membrane sector-associated protein of vacuolar proton-ATPase from chromaffin granules. J Biol Chem 273, 10939-10947.

12. Burckle, C., and Bader, M. (2006). Prorenin and its ancient receptor. Hypertension 48, 549551.

13. L'Huillier, N., Sharp, M.G.F., Dunbar, D.R., and Mullins, J.J. (2005). On the relationship between the renin receptor and the vacuolar proton ATPase membrane sector associated protein (M8-9). In: The Local Cardiac Renin Angiotensin-Aldosterone System, edited by Frolich ED and Re RN, New York, Springer, 2005: 17-34.

14. Forgac, M. (2007). Vacuolar ATPases: rotary proton pumps in physiology and pathophysiology. Nature reviews 8, 917-929.

15. Marshansky, V., and Futai, M. (2008). The V-type H+-ATPase in vesicular trafficking: targeting, regulation and function. Curr Opin Cell Biol 20, 415-426.

16. Beyenbach, K.W., and Wieczorek, H. (2006). The V-type H+ ATPase: molecular structure and function, physiological roles and regulation. The Journal of experimental biology 209, 577589.

17. Sihn, G., Rousselle, A., Vilianovitch, L., Burckle, C., and Bader, M. (2010). Physiology of the (pro)renin receptor: Wnt of change? Kidney Int 78, 246-256.

18. Schefe, J.H., Menk, M., Reinemund, J., Effertz, K., Hobbs, R.M., Pandolfi, P.P., Ruiz, P., Unger, T., and Funke-Kaiser, H. (2006). A novel signal transduction cascade involving direct physical interaction of the renin/prorenin receptor with the transcription factor promyelocytic zinc finger protein. Circ Res 99, 1355-1366. 
19. Kato, T., Du, D., Suzuki, F., and Park, E.Y. (2009). Localization of human (pro)renin receptor lacking the transmembrane domain on budded baculovirus of Autographa californica multiple nucleopolyhedrovirus. Appl Microbiol Biotechnol 82, 431-437.

20. Advani, A., Kelly, D.J., Cox, A.J., White, K.E., Advani, S.L., Thai, K., Connelly, K.A., Yuen, D., Trogadis, J., Herzenberg, A.M., et al. (2009). The (Pro)renin receptor: site-specific and functional linkage to the vacuolar H+-ATPase in the kidney. Hypertension 54, 261-269.

21. Amsterdam, A., Nissen, R.M., Sun, Z., Swindell, E.C., Farrington, S., and Hopkins, N. (2004). Identification of 315 genes essential for early zebrafish development. Proc Natl Acad Sci U S A $101,12792-12797$.

22. Nuckels, R.J., Ng, A., Darland, T., and Gross, J.M. (2009). The vacuolar-ATPase complex regulates retinoblast proliferation and survival, photoreceptor morphogenesis, and pigmentation in the zebrafish eye. Invest Ophthalmol Vis Sci 50, 893-905.

23. EauClaire, S.F., Cui, S., Ma, L., Matous, J., Marlow, F.L., Gupta, T., Burgess, H.A., Abrams, E.W., Kapp, L.D., Granato, M., et al. (2012). Mutations in vacuolar H+ -ATPase subunits lead to biliary developmental defects in zebrafish. Developmental biology 365, 434-444.

24. Gross, J.M., Perkins, B.D., Amsterdam, A., Egana, A., Darland, T., Matsui, J.I., Sciascia, S., Hopkins, N., and Dowling, J.E. (2005). Identification of zebrafish insertional mutants with defects in visual system development and function. Genetics 170, 245-261.

25. Wang, H., Kesinger, J.W., Zhou, Q., Wren, J.D., Martin, G., Turner, S., Tang, Y., Frank, M.B., and Centola, M. (2008). Identification and characterization of zebrafish ocular formation genes. Genome / National Research Council Canada = Genome / Conseil national de recherches Canada 51, 222-235.

26. Cruciat, C.M., Ohkawara, B., Acebron, S.P., Karaulanov, E., Reinhard, C., Ingelfinger, D., Boutros, M., and Niehrs, C. (2010). Requirement of prorenin receptor and vacuolar $\mathrm{H+}$ ATPase-mediated acidification for Wnt signaling. Science (New York, N.Y 327, 459-463.

27. Clevers, H., and Nusse, R. (2012). Wnt/beta-catenin signaling and disease. Cell 149, 11921205.

28. Niehrs, C. (2012). The complex world of WNT receptor signalling. Nature reviews 13, 767779.

29. Bilic, J., Huang, Y.L., Davidson, G., Zimmermann, T., Cruciat, C.M., Bienz, M., and Niehrs, C. (2007). Wnt induces LRP6 signalosomes and promotes dishevelled-dependent LRP6 phosphorylation. Science (New York, N.Y 316, 1619-1622.

30. Blitzer, J.T., and Nusse, R. (2006). A critical role for endocytosis in Wnt signaling. BMC cell biology 7, 28.

31. Yamamoto, H., Komekado, H., and Kikuchi, A. (2006). Caveolin is necessary for Wnt-3adependent internalization of LRP6 and accumulation of beta-catenin. Developmental cell 11, 213-223.

32. Bartscherer, K., Pelte, N., Ingelfinger, D., and Boutros, M. (2006). Secretion of Wnt ligands requires Evi, a conserved transmembrane protein. Cell 125, 523-533.

33. Mummery-Widmer, J.L., Yamazaki, M., Stoeger, T., Novatchkova, M., Bhalerao, S., Chen, D., Dietzl, G., Dickson, B.J., and Knoblich, J.A. (2009). Genome-wide analysis of Notch signalling in Drosophila by transgenic RNAi. Nature 458, 987-992.

34. Allan, A.K., Du, J., Davies, S.A., and Dow, J.A. (2005). Genome-wide survey of V-ATPase genes in Drosophila reveals a conserved renal phenotype for lethal alleles. Physiological genomics 22, 128-138.

35. Buechling, T., Bartscherer, K., Ohkawara, B., Chaudhary, V., Spirohn, K., Niehrs, C., and Boutros, M. (2010). Wnt/Frizzled signaling requires dPRR, the Drosophila homolog of the prorenin receptor. Curr Biol 20, 1263-1268.

36. Hermle, T., Saltukoglu, D., Grunewald, J., Walz, G., and Simons, M. (2010). Regulation of Frizzled-dependent planar polarity signaling by a V-ATPase subunit. Curr Biol 20, 1269-1276.

37. Hermle, T., Guida, M.C., Beck, S., Helmstadter, S., and Simons, M. (2013). Drosophila ATP6AP2/VhaPRR functions both as a novel planar cell polarity core protein and a regulator of endosomal trafficking. The EMBO journal 32, 245-259. 
38. Bader, M., Peters, J., Baltatu, O., Muller, D.N., Luft, F.C., and Ganten, D. (2001). Tissue reninangiotensin systems: new insights from experimental animal models in hypertension research. Journal of molecular medicine (Berlin, Germany) 79, 76-102.

39. Mizushima, N. (2007). Autophagy: process and function. Genes Dev 21, 2861-2873.

40. Levine, B., Mizushima, N., and Virgin, H.W. (2011). Autophagy in immunity and inflammation. Nature 469, 323-335.

41. Kinouchi, K., Ichihara, A., Sano, M., Sun-Wada, G.H., Wada, Y., Kurauchi-Mito, A., Bokuda, K., Narita, T., Oshima, Y., Sakoda, M., et al. (2010). The (pro)renin receptor/ATP6AP2 is essential for vacuolar H+-ATPase assembly in murine cardiomyocytes. Circ Res 107, 30-34.

42. Riediger, F., Quack, I., Qadri, F., Hartleben, B., Park, J.K., Potthoff, S.A., Sohn, D., Sihn, G., Rousselle, A., Fokuhl, V., et al. (2011). Prorenin receptor is essential for podocyte autophagy and survival. J Am Soc Nephrol 22, 2193-2202.

43. Oshima, Y., Kinouchi, K., Ichihara, A., Sakoda, M., Kurauchi-Mito, A., Bokuda, K., Narita, T., Kurosawa, H., Sun-Wada, G.H., Wada, Y., et al. (2011). Prorenin receptor is essential for normal podocyte structure and function. J Am Soc Nephrol 22, 2203-2212.

44. Hirata, R., Umemoto, N., Ho, M.N., Ohya, Y., Stevens, T.H., and Anraku, Y. (1993). VMA12 is essential for assembly of the vacuolar $\mathrm{H}(+)$-ATPase subunits onto the vacuolar membrane in Saccharomyces cerevisiae. J Biol Chem 268, 961-967.

45. Graham, L.A., Powell, B., and Stevens, T.H. (2000). Composition and assembly of the yeast vacuolar $\mathrm{H}(+)$-ATPase complex. The Journal of experimental biology 203, 61-70.

46. Hirose, T., Hashimoto, M., Totsune, K., Metoki, H., Asayama, K., Kikuya, M., Sugimoto, K., Katsuya, T., Ohkubo, T., Hashimoto, J., et al. (2009). Association of (pro)renin receptor gene polymorphism with blood pressure in Japanese men: the Ohasama study. Am J Hypertens 22, 294-299.

47. Ott, C., Schneider, M.P., Delles, C., Schlaich, M.P., Hilgers, K.F., and Schmieder, R.E. (2011). Association of (pro)renin receptor gene polymorphism with blood pressure in Caucasian men. Pharmacogenetics and genomics 21, 347-349.

48. Hirose, T., Hashimoto, M., Totsune, K., Metoki, H., Hara, A., Satoh, M., Kikuya, M., Ohkubo, T., Asayama, K., Kondo, T., et al. (2011). Association of (pro)renin receptor gene polymorphisms with lacunar infarction and left ventricular hypertrophy in Japanese women: the Ohasama study. Hypertens Res 34, 530-535.

49. Brugts, J.J., Isaacs, A., de Maat, M.P., Boersma, E., van Duijn, C.M., Akkerhuis, K.M., Uitterlinden, A.G., Witteman, J.C., Cambien, F., Ceconi, C., et al. (2011). A pharmacogenetic analysis of determinants of hypertension and blood pressure response to angiotensinconverting enzyme inhibitor therapy in patients with vascular disease and healthy individuals. Journal of hypertension 29, 509-519.

50. Ramser, J., Abidi, F.E., Burckle, C.A., Lenski, C., Toriello, H., Wen, G., Lubs, H.A., Engert, S., Stevenson, R.E., Meindl, A., et al. (2005). A unique exonic splice enhancer mutation in a family with $X$-linked mental retardation and epilepsy points to a novel role of the renin receptor. Human molecular genetics 14, 1019-1027.

51. Korvatska, O., Strand, N.S., Berndt, J.D., Strovas, T., Chen, D.H., Leverenz, J.B., Kiianitsa, K., Mata, I.F., Karakoc, E., Greenup, J.L., et al. (2013). Altered splicing of ATP6AP2 causes Xlinked parkinsonism with spasticity (XPDS). Human molecular genetics.

52. Poorkaj, P., Raskind, W.H., Leverenz, J.B., Matsushita, M., Zabetian, C.P., Samii, A., Kim, S., Gazi, N., Nutt, J.G., Wolff, J., et al. (2010). A novel X-linked four-repeat tauopathy with Parkinsonism and spasticity. Mov Disord 25, 1409-1417.

53. Contrepas, A., Walker, J., Koulakoff, A., Franek, K.J., Qadri, F., Giaume, C., Corvol, P., Schwartz, C.E., and Nguyen, G. (2009). A role of the (pro)renin receptor in neuronal cell differentiation. Am J Physiol Regul Integr Comp Physiol 297, R250-257.

54. Takamori, S., Holt, M., Stenius, K., Lemke, E.A., Gronborg, M., Riedel, D., Urlaub, H., Schenck, S., Brugger, B., Ringler, P., et al. (2006). Molecular anatomy of a trafficking organelle. Cell 127, 831-846. 
55. Piper, R.C., and Katzmann, D.J. (2007). Biogenesis and function of multivesicular bodies. Annual review of cell and developmental biology 23, 519-547.

56. Taelman, V.F., Dobrowolski, R., Plouhinec, J.L., Fuentealba, L.C., Vorwald, P.P., Gumper, I., Sabatini, D.D., and De Robertis, E.M. (2010). Wnt signaling requires sequestration of glycogen synthase kinase 3 inside multivesicular endosomes. Cell 143, 1136-1148.

57. Gross, J.C., Chaudhary, V., Bartscherer, K., and Boutros, M. (2012). Active Wnt proteins are secreted on exosomes. Nature cell biology 14, 1036-1045.

58. Liegeois, S., Benedetto, A., Garnier, J.M., Schwab, Y., and Labouesse, M. (2006). The V0ATPase mediates apical secretion of exosomes containing Hedgehog-related proteins in Caenorhabditis elegans. The Journal of cell biology 173, 949-961.

59. Williamson, W.R., Wang, D., Haberman, A.S., and Hiesinger, P.R. (2010). A dual function of V0-ATPase a1 provides an endolysosomal degradation mechanism in Drosophila melanogaster photoreceptors. The Journal of cell biology 189, 885-899.

60. Hiesinger, P.R., Fayyazuddin, A., Mehta, S.Q., Rosenmund, T., Schulze, K.L., Zhai, R.G., Verstreken, P., Cao, Y., Zhou, Y., Kunz, J., et al. (2005). The v-ATPase V0 subunit a1 is required for a late step in synaptic vesicle exocytosis in Drosophila. Cell 121, 607-620.

61. Lee, S.H., Rho, J., Jeong, D., Sul, J.Y., Kim, T., Kim, N., Kang, J.S., Miyamoto, T., Suda, T., Lee, S.K., et al. (2006). v-ATPase V0 subunit d2-deficient mice exhibit impaired osteoclast fusion and increased bone formation. Nature medicine 12, 1403-1409.

62. Peri, F., and Nusslein-Volhard, C. (2008). Live imaging of neuronal degradation by microglia reveals a role for v0-ATPase a1 in phagosomal fusion in vivo. Cell 133, 916-927.

63. Strasser, B., Iwaszkiewicz, J., Michielin, O., and Mayer, A. (2011). The V-ATPase proteolipid cylinder promotes the lipid-mixing stage of SNARE-dependent fusion of yeast vacuoles. The EMBO journal 30, 4126-4141.

64. Maruyama, N., Segawa, T., Kinoshita, N., and Ichihara, A. (2010). Novel sandwich ELISA for detecting the human soluble (pro)renin receptor. Frontiers in bioscience (Elite edition) 5 , 583-590.

65. Lu, X., Garrelds, I.M., Wagner, C.A., Danser, A.H., and Meima, M.E. (2013). (Pro)renin Receptor is Required for Prorenin-Dependent and -Independent Regulation of Vacuolar H+ATPase Activity in MDCK.C11 Collecting Duct Cells. American journal of physiology. 


\section{FIGURE LEGENDS}

Figure 1: Timeline of $\mathbf{( P ) R R}$ characterization. The timeline summarizes the different studies describing an association between (P)RR and V-ATPase. For references see text. PCP, planar cell polarity; (P)RR, (pro)renin receptor; XLMR, X-linked associated mental retardation; XPDS, X-linked Parkinsonism with spasticity.

Figure 2: Structure and potential binding partners of (P)RR. Proteins from various Wnt signaling pathways and from the V-ATPase complex have been shown to interact (directly or indirectly) with (P)RR. For references see text. Fmi, Flamingo; Fz, Frizzled; LRP6, lowdensity lipoprotein receptor-related protein 6; PCP, planar cell polarity; (P)RR, (pro)renin receptor; RAS, renin-angiotensin system; V-ATPase, vacuolar $\mathrm{H}^{+}$ATPase.

Figure 3: Functions of (P)RR. In Xenopus, (P)RR was shown to be important for canonical Wnt signling. In mice, a role of (P)RR in the regulation of autophagy was described. In Drosophila, (P)RR was essential for PCP core protein trafficking and for endocytosis/recycling of apical proteins. For references see text. E-Cad, E-cadherin; ER, endoplasmic reticulum; Fmi, Flamingo; Fz, Frizzled; LRP6, low-density lipoprotein receptorrelated protein 6; PCP, planar cell polarity; (P)RR, (pro)renin receptor; V-ATPase, vacuolar $\mathrm{H}^{+}$ATPase. 


\section{FIGURES}

\section{Figure 1}

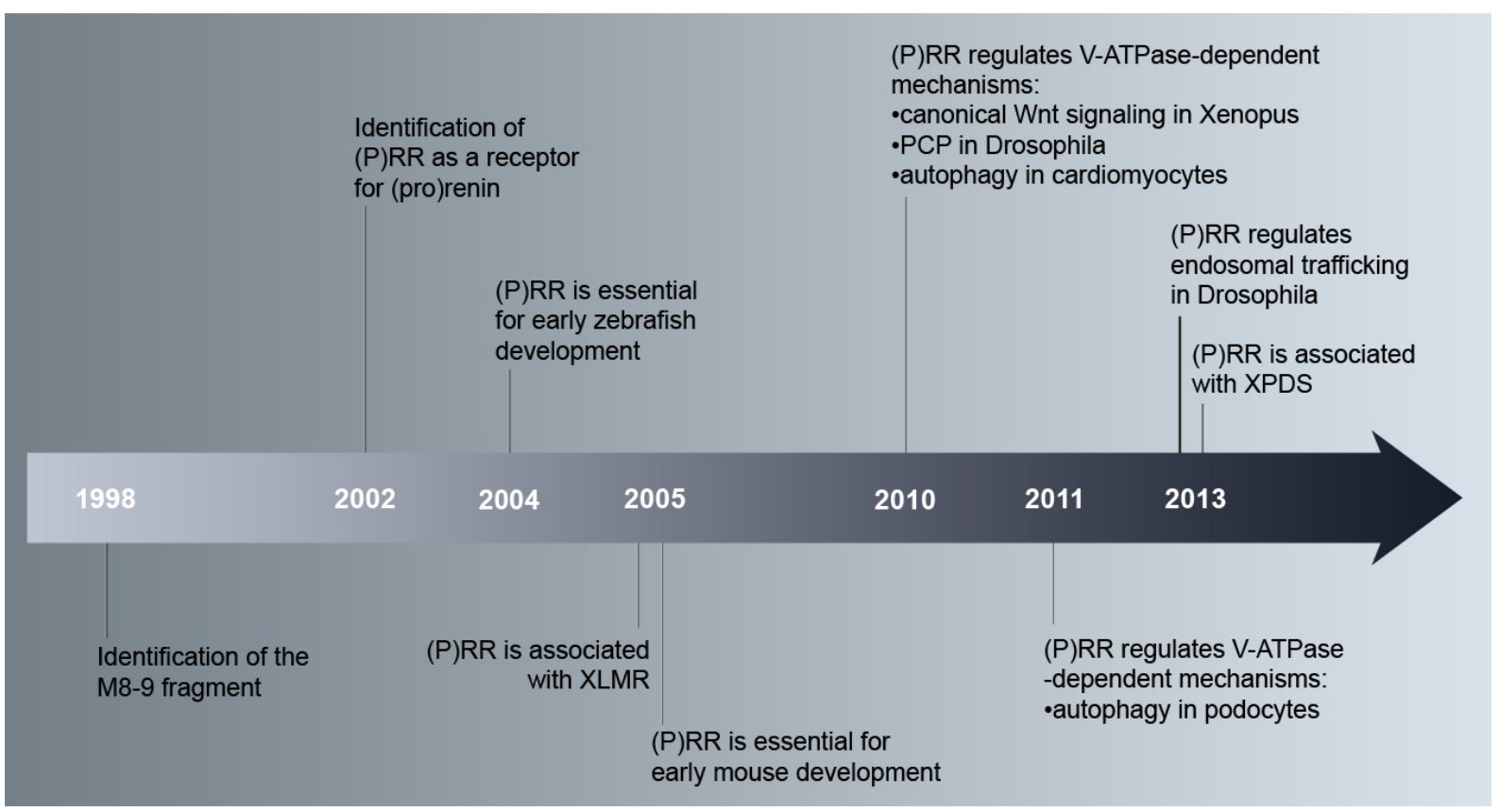


Figure 2

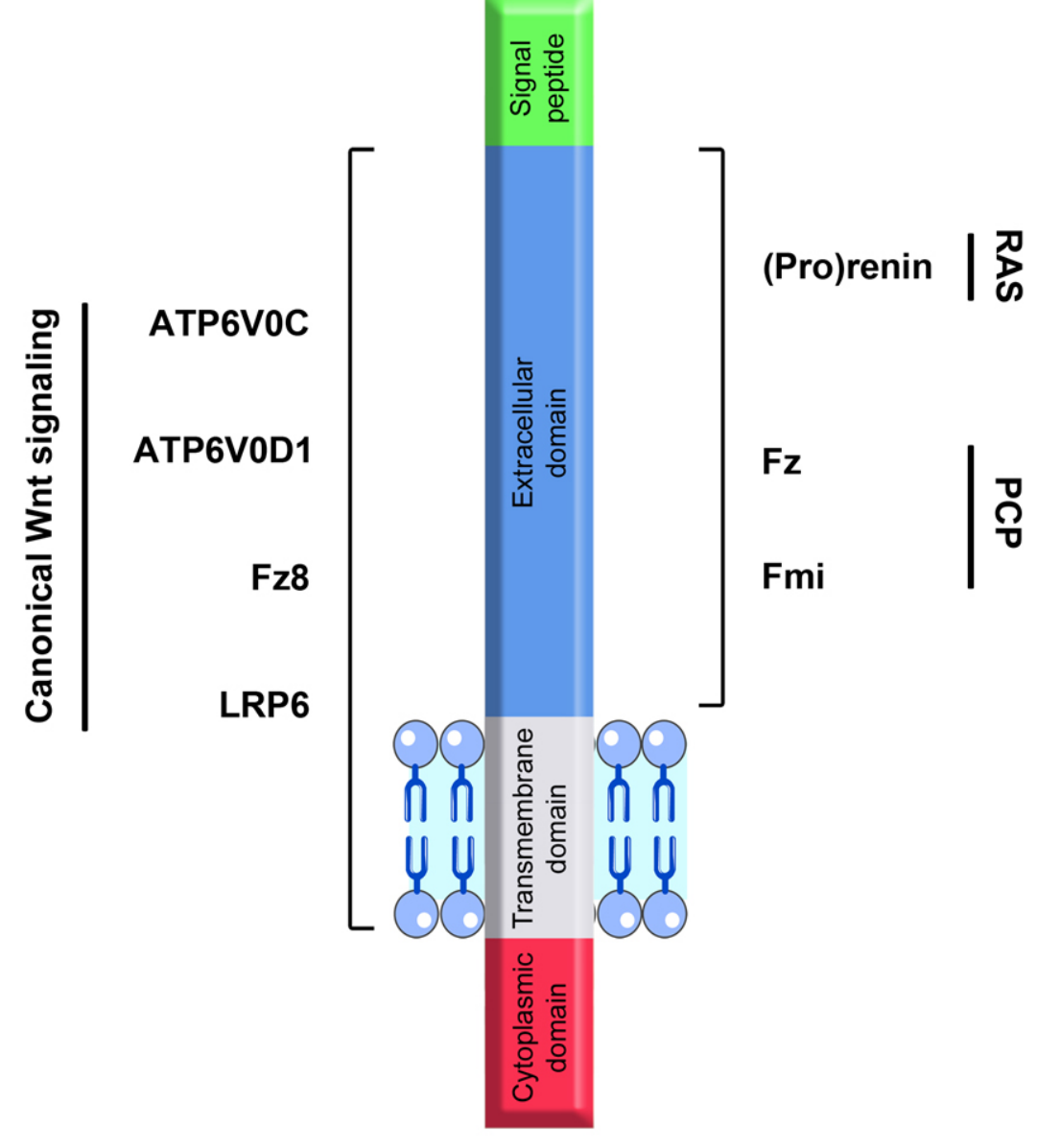


Figure 3

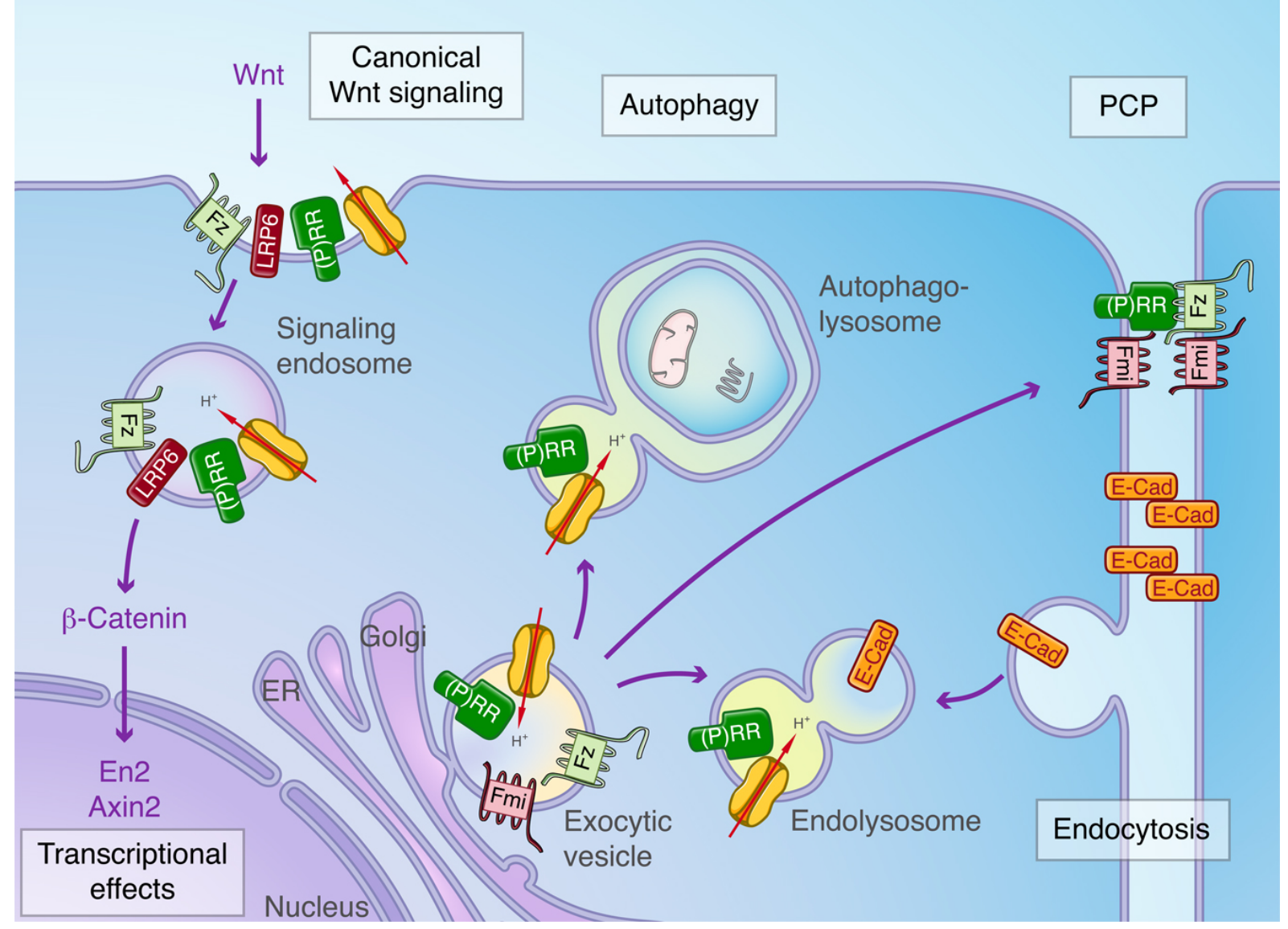

